\title{
Surface enhanced Infrared Absorption in dielectric thin films with ultra-strong confinement effects
}

\author{
Yann Chalopin, 1 , * Marc Hayoun, ${ }^{2}$ Sebastian Volz, ${ }^{1}$ and Hichem Dammak ${ }^{3,2}$ \\ ${ }^{1}$ Laboratoire d'Energétique Moléculaire et Macroscopique, CNRS UPR 288, \\ Ecole Centrale Paris, F-92295 Châtenay-Malabry, France \\ ${ }^{2}$ Laboratoire des Solides Irradis, Ecole Polytechnique, CEA-DSM, CNRS, F-91128 Palaiseau, France \\ ${ }^{3}$ Laboratoire Structure Proprits et Modlisation des Solides, \\ CNRS UMR 8580, Ecole Centrale Paris, F-92295 Châtenay-Malabry, France
}

\begin{abstract}
By formulating a microscopic description of the non-local dielectric constant, we have investigated the mechanisms of infrared absorption in dielectrics thin films by molecular dynamics simulations. We found that light absorption in dielectric slabs does not occur predominantly at the polaritons resonances but through anomalous surface modes extremely confined in space. This demonstrate that any macroscopic description of electrodynamics in dielectrics breaks down at the nanoscale.
\end{abstract}

The study of the optical properties of nano-materials involves two main classes of physical systems: metals and dielectrics. At the nanoscale, both exhibit particularly interesting properties due to the resonances activated by the confining boundary conditions - e.g surface phononpolariton for dielectrics and surface plasmon for metals. Understanding the microscopic mechanisms of light coupling with nanomaterials forms the cornerstone of the fields of nanophotonics and nano-optics from which a large panel of applications have recently emerged such as the enhancements of Raman scattering $\stackrel{1}{1}$, the fluorescence imaging ${ }^{2}$ or the control of coherent thermal radiation. When studying how size-confinement impacts optical properties at the short-scales, it has been reported that non-local effects take place in metals 4.5 such as the spill-out of electrons $\frac{6.7}{}$ : For systems smaller than $10 \mathrm{~nm}$, a strong increase of the intrinsic electron-phonon interaction can be observed. On the other hand, very few work reported peculiar size effects in dielectric materials $\frac{9}{}$ although there has been much effort devoted to exploring optical modes of vibration in these systems 12,21 .

In this paper, the microscopic mechanisms of IR-light absorption in a model $(\mathrm{MgO})$ of confined dielectric materials of few atomic layers thickness are investigated. In these systems, it is notably demonstrated that the optical response in the $\mathrm{THz}$ regime exhibits anomalous size effects indicating that any macroscopic description of infra-red (IR) light/matter interaction breaks down at the atomic scale. A microscopic non-local model that allows the recovery of the frequency versus wave-vector polarizability spectra is presented. It is based on the tracking of the equilibrium polarization fluctuations. In this study, the IR absorption spectra for $\mathrm{MgO}$ slabs thickness ranging from 0.4 to $4 \mathrm{~nm}$ which are shown to be size dependent. Ultra strong confined modes are unveiled and their physical origin are discussed.

To this aim, a microscopic formalism that captures

\footnotetext{
a Applied Physics Letter, 104(2014)011905. DOI: $10.1063 / 1.4860989$
}

the non-local dielectric constant is developed. It is adequate to equilibrium molecular dynamics (MD) simulations. Our approach is formulated on the basis of the linear response theory. The susceptibility $\chi$ of a system subjected to an external electric field $\mathbf{E}$ can be expressed as

$$
p_{\beta}(\omega, \mathbf{k})=V \varepsilon_{0} \chi_{\beta \gamma}(\omega, \mathbf{k}) E_{\gamma}(\omega, \mathbf{k}),
$$

with $\mathbf{p}$ the dipolar moment induced by $\mathbf{E}$. The indexes $\beta$ and $\gamma$ stand for the space coordinates $(x, y, z)$ and $V$ is the volume of the dielectric system. For any anisotropic materials, the fluctuation-dissipation theorem 13 allows to generally express the tensor $\chi$ as ${ }^{14}$

$$
\chi_{\beta \gamma}(\omega, \mathbf{k})=\frac{1}{V \varepsilon_{0} k_{B} T}\left[\left\langle p_{\beta}(0, \mathbf{k}) p_{\gamma}(0, \mathbf{k})\right\rangle+i \omega \int_{0}^{\infty} e^{i \omega t}\left\langle p_{\beta}(0, \mathbf{k}) p_{\gamma}(\right.\right.
$$

where $\mathbf{p}$ is expressed in the reciprocal space as

$$
p_{\beta}(t, \mathbf{k})=\sum_{j} q_{j} \cdot r_{j \beta(t)} e^{i \mathbf{k} \cdot \mathbf{r}_{j}^{0}},
$$

$\mathbf{r}_{j}^{0}$ is the equilibrium position of atom $j$ caring the charge $q_{j}$. Hence, the optical spectra of any dielectric material are extracted from the fluctuations of the system at thermal equilibrium and the non-local description of their dielectric properties corresponds to the Fourier transform of the charges fluctuations. For a bulk material, the dielectric constant $\varepsilon$ is complex, $\varepsilon=\varepsilon^{\prime}+i \varepsilon^{\prime \prime}$, and one has:

$$
\begin{gathered}
\varepsilon^{\prime}=\operatorname{Re}\{\chi\}+1 \\
\varepsilon^{\prime \prime}=\operatorname{Im}\{\chi\}
\end{gathered}
$$

For a dielectric slab:

$$
V \chi=\alpha=\alpha^{\prime}+i \alpha^{\prime \prime}
$$

$\alpha$ is the polarizability.

MD simulations were performed by using an empirical potential $^{15}$ which has been demonstrated to be valid for 
bulk and surface properties 16,17 . We have first studied the $\mathrm{MgO}$ bulk with a crystal of 8000 atoms which corresponds to a $10 \times 10 \times 10$ cubic cell $\left(10^{3}\right.$ points in the first Brillouin zone) with periodic boundary conditions.

We have validated our approach by recovering the dielectric constant at $\mathbf{k}=0$ to allow a fair comparison with the available experimental data ${ }^{18}$ (see Fig [1). We have calculated the non-local imaginary part of the dielectric constant, $\epsilon^{\prime \prime}(\omega, \mathbf{k})$, with $\mathbf{k}$ ranging along the high symmetry lines of the first Brillouin zone (Fig. 1). From these two plots, we can see that the bulk absorption at the long wavelength limit corresponds to the TO mode at the frequency of $f_{T O}=12 \mathrm{THz}$. In contrast, we can see that for $\mathbf{k}=\mathbf{0}$, the polar $\mathrm{LO}(25 \mathrm{THz})$ is absent (Fig. 1,a).

We now consider the case of a crystal slab of finite thickness $L=3 a$ in the $z$ direction [001] and periodic in the two lateral ones $x, y$ ([100] and [010]). The in-plane modes are defined by the wave-vector components $\left(k_{x}, k_{y}\right)$ and the cross plane one by $k_{z}$ as $\alpha_{\beta \gamma}\left(k_{x}, k_{y}, k_{z}, \omega\right)$. The absorption at long wavelength limit $\left(k_{x}=k_{y}=k_{z}=0\right)$ is given on Figure 2 a). It can be seen that IR absorption occurs through mainly two bands at 12 and $25 \mathrm{THz}$.

To elucidate the physical origin of these IR resonances which are not observed in the bulk, the spatial mapping of the absorption processes has been performed at each resonant frequency (Figure 2 middle). To this aim, each atom $i$ of charge $q_{i}$ has been subjected to a polarized electric force $q_{i} \mathbf{E}$ and the corresponding local absorption, $a_{i}$, has been traced back with the local dipolar moment $q_{i} x_{i}$ as

$$
a_{i}^{\|, \perp}(\omega)=\frac{\omega}{k_{B} T} \int_{0}^{\infty} q_{i}^{2}\left\langle\mathbf{x}_{i}^{\|, \perp}(0) \mathbf{x}_{i}^{\|, \perp}(t)\right\rangle e^{i \omega t} d t,
$$

where $\mathbf{x}_{i}^{\|, \perp}$ refers to the displacement of atom $i$ in the cross plane $(\perp)$ or in the in-plane directions $(\|)$ of the slab.

The lowest frequency band at $12 \mathrm{THz}$ corresponds to in-plane polarization ([100], [010]) whereas absorption at the highest frequency band $25 \mathrm{THz}$ corresponds to cross plane polarization $([001])$. It can be seen that the polar mode at the frequency $f_{1}$ involves surface atoms whereas $f_{2}$ and $f_{3}$ corresponds to volume modes. This clearly indicates that in this slab and more generally suggests that in dielectric thin films, IR light is absorbed at the bulk TO and $\mathrm{LO}^{24}$ frequencies in addition to a surface mode. Interestingly, the absorption occurs predominantly through this surface mode $\left(f_{1}\right)$ which is completely localized at the topmost layers. The modes $f_{2}$ and $f_{3}$ are the transverse and longitudinal polaritons, respectively. The $f_{1}$ and $f_{3}$ modes originate from the finite boundary conditions of the slab. The appearance of the $f_{3}$ mode is a clear evidence of the Berreman effect ${ }^{25}$. We think that a signature of this mode can be possibly observed experimentally 18 even while measuring the bulk dielectric constant (see Figure 1 at $28 \mathrm{THz}$ ) as the mea- surements always involve a dielectric/air interface at a surface of the crystal sample.

The non-local polarizability calculated in the first Brillouin zone (Fig. 2 bottom) shows that the LO and TO polaritons have dispersion relations similar to those of the bulk LO and TO branches. On another hand, it can be seen that the surface mode $\left(f_{1}\right)$ is non-dispersive along the $k_{z}$ direction. In contrast to the bulk non local properties, extra polar modes are activated at lowest frequencies from the $K$ point to the $\Gamma$ point.

To investigate the size effects, the optical spectra have been studied at different film thicknesses ranging from $L=1 a$ to $10 a$. The corresponding absorption spectra are presented on top of Figure 3. For the thinner thickness ( $L=1 a$; two planes), it is observed two peaks at frequencies of 11 and $23 \mathrm{THZ}$, respectively. These two peaks correspond to polaritons modes involving the displacements of all the atoms parallelly and perpendicularly to the slab, respectively. Let us first consider the size dependence of the $11 \mathrm{THz}$ peak : When increasing the layer thickness from $\mathrm{L}=2 \mathrm{a}$, we observe a splitting of this mode into two contributions identified as $f_{1}$ and $f_{2}$. Such a splitting occurs because the topmost atomic layers undergo interatomic force constants weaker than that of the volume layers where the atoms are isotropically bonded. Below $\mathrm{L}=5 \mathrm{a}$, the absorption by the surface mode, $f_{1}$, predominates over the absorption of the TO polariton mode, $f_{2}$. Recalling that while increasing $\mathrm{L}$, the number of surface atoms get decreased with respect to the volume one, it can be noticed that even for a ratio of $20 \%$ at $L=5 a$, the absorption due to the topmost layers remains as important as that of the volume ones.

We observe two additional peaks between 20 and 25 $\mathrm{THz}$ with very much smaller amplitudes. Their associated frequencies and amplitudes are size dependent. They progressively disappear at the thicker thicknesses. By mapping the local polarisability (Equ 6 for $L=3$ a, we found that the absorption peak around $21 \mathrm{THz}$ involves the atoms of the planes underneath the outermost planes of the slab (not displayed). The peak around 23$25 \mathrm{THz}$ originates from the atoms of the third deepest surface planes. Thus, these two absorptions are very localized. The frequencies indicates that the physical origin of the additional peaks does not correspond to the modes with a wavelength $\lambda$ that fits the thickness $\mathrm{L}$ as $L=n \lambda / 2^{11,21}$. Indeed, the number of modes does not increase as the layer thickness increases. We attribute this effect to the dielectric screening by the topmost layers 9 .

By looking at the size dependence of the non-local polarisability (fig. 3-bottom) in the [001] direction (i.e along the slab thickness), we see that the TO absorption modes in the range $11-15 \mathrm{THz}$ corresponds to a competition between the polariton branch and the surface branch strongly localized at the topmost atomic layers. The surface branch remains non-dispersive whereas the dispersion of the polariton branch tends to that observed for the bulk (Fig. 1 bottom) as the thickness increases. In a similar fashion, the LO branch at higher frequen- 
cies $(20-25 \mathrm{THz})$ corresponds to the polariton mode and its dispersion converges to the bulk one at thicker thicknesses. In addition, its intensity (i.e the polarisability) is weaker than that of the TO modes for the various thicknesses. These discrepancies can be attributed to the effect of dielectric screening by the surface layers as it has been observed for the case of $\mathrm{MgO}$ nanoparticles of few nm $\operatorname{size}^{9}$.

To conclude, we have demonstrated that IR optical absorption in dielectric slabs of few nanometers is driven by strongly localized surface processes which are not predicted by a macroscopic description of electrodynamics. These peculiar resonances originating from the vibrational dynamics at the surfaces of finite thickness slabs enhance the radiation absorption in an important way and are predominant among other usual absorption processes (polaritons). To unveil the physical origin of these effects, we have presented a microscopic non-local model based on molecular dynamics simulations and which provides a convenient framework for investigating optical properties of matter at the nanoscale.
* Corresponding author: yann.chalopin@ecp.fr

1 S. Nie and S.R. Emory, Science 275, 1102 (1997).

${ }^{2}$ F. Shafiei, C. Wu, Y. Wu, A. B. Khanikaev, P. Putzke, A. Singh, X. Li, G. Shvets, Nature Photonics 7, 367 (2013).

3 J.-J. Greffet, R. Carminati, K . Joulain, J. P. Mulet, S. Mainguy, Y. Chen, Nature 416, 61 (2002).

4 J. M. McMahon, Stephen K. G., and G. C. Schatz Rev. Lett. 103, 097403 (2009). J. Phys. Chem. C 115, 19470 (2011)

${ }^{5}$ C. David and F. J. Garca de Abajo, J. Phys. Chem. C, 115, 194701719475 (2011).

${ }^{6}$ C. Voisin, N. Del Fatti, D. Christofilos, F. Vallée, J. Phys. Chem. B 105, 2264 (2001).

7 J. B. Gaudry, L. Capes, P. Langot, S. Marcen, M. Kollmannsberger, O. Lavastre, E. Freysz, J. F. Letard, and O. Kahn, Chem. Phys. Lett. 324, 321 (2000).

${ }^{8}$ H., Raether, Surface polariton (Springer, New York, 1988).

9 Y. Chalopin, H. Dammak, M. Hayoun, M. Besbes, J. J. Greffet, App. Phys. Lett. 100 (2012).

10 W. E. Jones, R. Fuchs, Phys. Rev. B, 4, 3581,(1971).

11 K. L. Kliewer and Ronald Fuchs, Phys. Rev. 150, 573 (1966).

12 S. Y. Tong and A. A. Maradudin, Phys. Rev. 181, 1318 (1969).

13 R Kubo 1966 Rep. Prog. Phys. 29, 255, (1966).

14 Y. Chalopin, H. Dammak, M. Laroche, M. Hayoun, and
J.J. Greffet, Phys. Rev. B 84, 224301 (2011).

15 M. Matsui, J.Chem.Phys. 91, 489 (1989).

16 G. Geneste, M. Hayoun, F. Finocchi, and J. Morillo, J. Phys.: Condens. Matter, 21, 315004 (2009).

17 E. Antoshchenkova, M. Hayoun, G. Geneste, and F. Finocchi, Phys. Chem. Chem. Phys. 12, 7251, (2010).

18 E. D. Palik and J. M. Bennett, Handbook of Optical Constants of Solids, vol II. (Academic Press, New York, 1991).

19 G. Scarel, G. K. Hyde, D. Hojo, and G. N. Parsons, J. Appl. Phys. 104, 094314 (2008).

20 R. B. Barnes and M. Czerny, Z. Physik 72, 447 (1931)

${ }^{21}$ G. O. Jones, D. H. Martin, P. A. Mawer, and C. H. Perry, Proc. Roy. Soc. (London) A261, 10 (1961).

${ }^{22}$ W. Cochran, S. J. Fray, F. A. Johnson, J. E. Quarrington, and N. Williams, Proc. Phys. Soc. (London) 77, 215 (1961)

${ }^{23}$ S. J. Fray, F. A. Johnson, J. E. Quarrington, and N. Williams, J. Appl. Phys. 32, 2102 (1961).

${ }^{24}$ X-Q. Li, Y. Arakawa, Sol. State Comm. 118, 211, (1998).

${ }^{25}$ D. W. Berreman, Phys. Rev. 130, 2193172198 (1963).

26 T. S. Chen, G. P. Alldredge, and F. W. de Wette, Phys. Rev. B, 6, 15, (1972).

27 A. A. Lucas, J. Chem. Phys. 48, 3156 (1968).

28 S. Vassant, J. P. Hugonin, F. Marquier, J. J. Greffet, Optics Express, 20, 23971, (2012)

29 E. A. Vinogradov, G. N. Zhizhin, A. G. Mal'shukov, Sov. Phys., JETP 48, 778, (1977). 
Dielectric constant $\varepsilon$
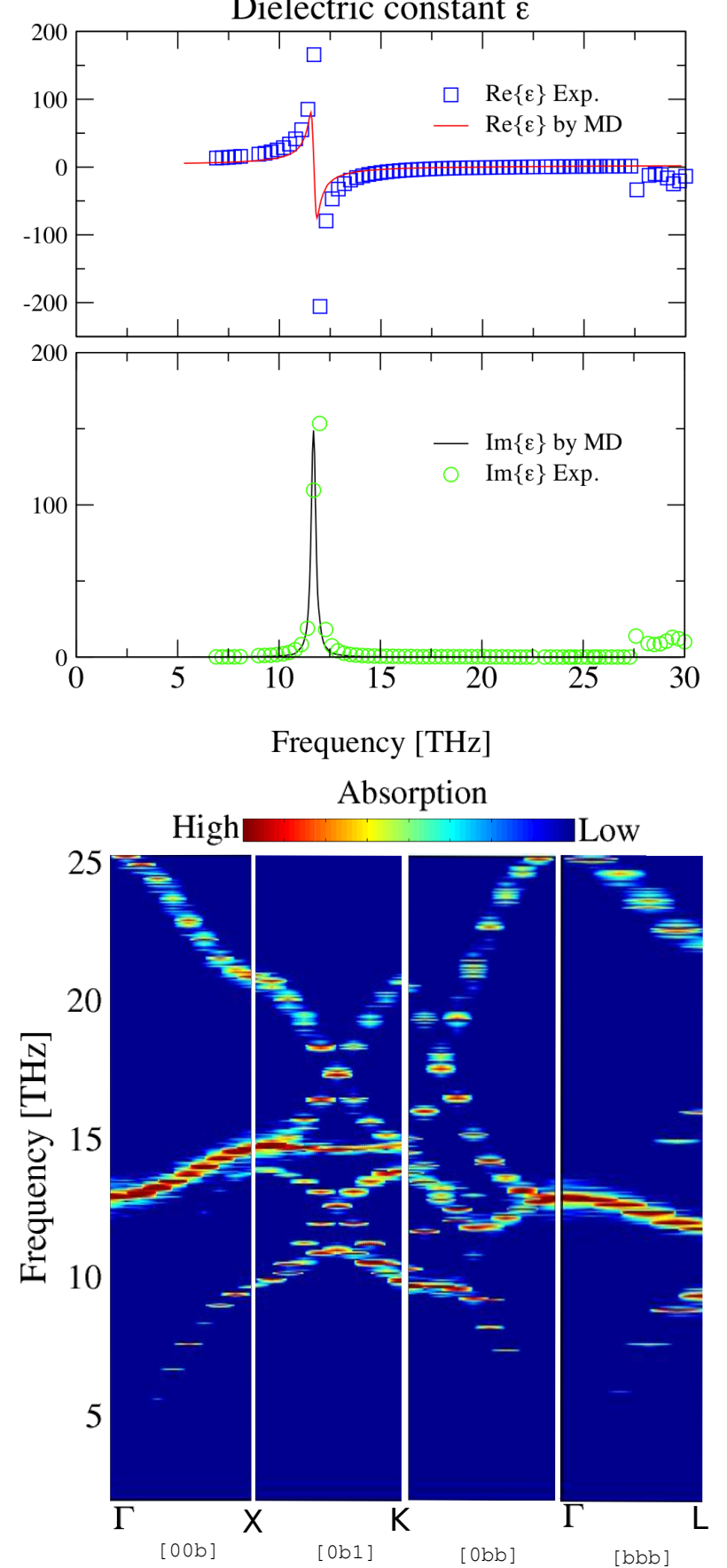

FIG. 1. Top: frequency dependent bulk dielectric constant $\left(\left(\epsilon_{x x}+\epsilon_{y y}+\epsilon_{z z}\right) / 3\right)$ compared to experimental data ${ }^{18}$; Bottom: the corresponding non-local spectra $(\operatorname{Tr}[\epsilon(\omega, \mathbf{k}])$ in $\log$ scale) along the main directions of the $\mathrm{MgO}$ first Brillouin zone. 

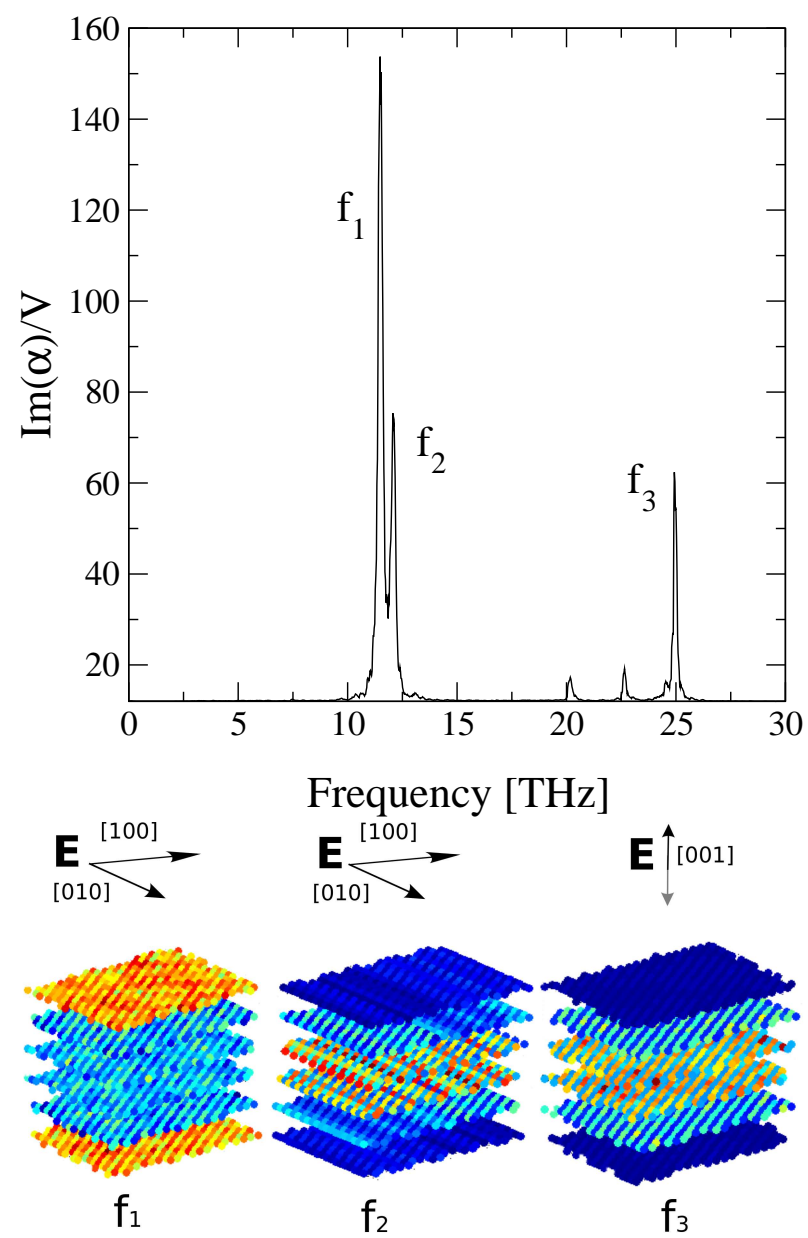

Absorption

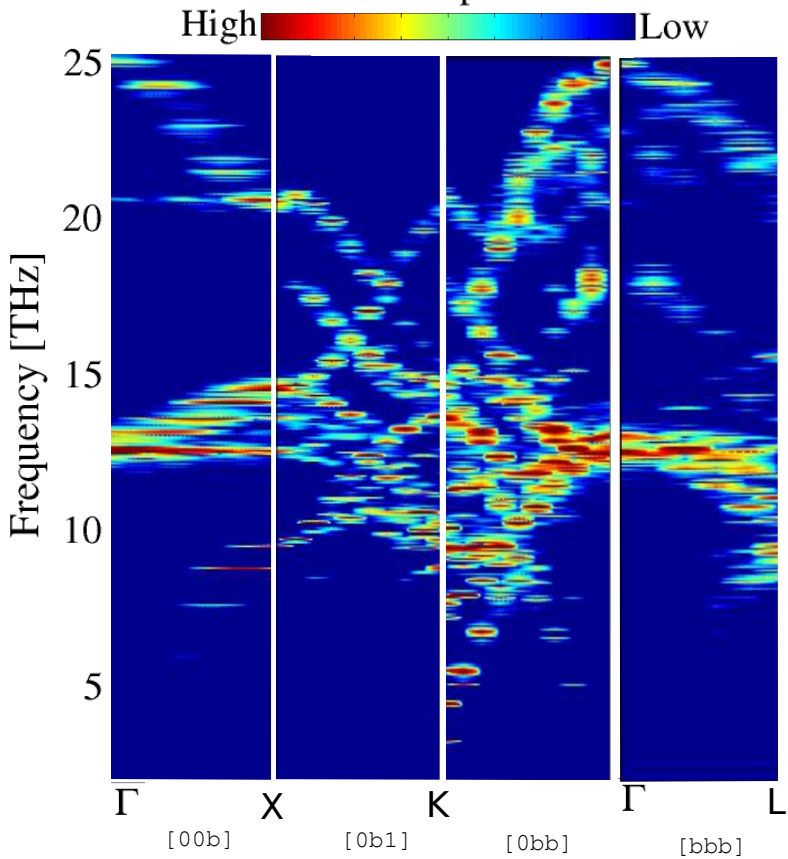

FIG. 2. Thickness $L=3 a$. Top : Imaginary part of the slab polarisability $\left(\left(\alpha_{x x}^{\prime \prime}+\alpha^{\prime \prime} y y+\alpha^{\prime \prime} z z\right) / 3\right)$ at $\mathbf{k}=0$; Middle: mapping of the absorption for each atoms (eq. 6) at the three dominant absorption frequencies $f_{1}, f_{2}$ and $f_{3}$; Bottom: $\operatorname{Tr}\left[\alpha^{\prime \prime}(\omega, \mathbf{k})\right]$ in $\log$ scale. 

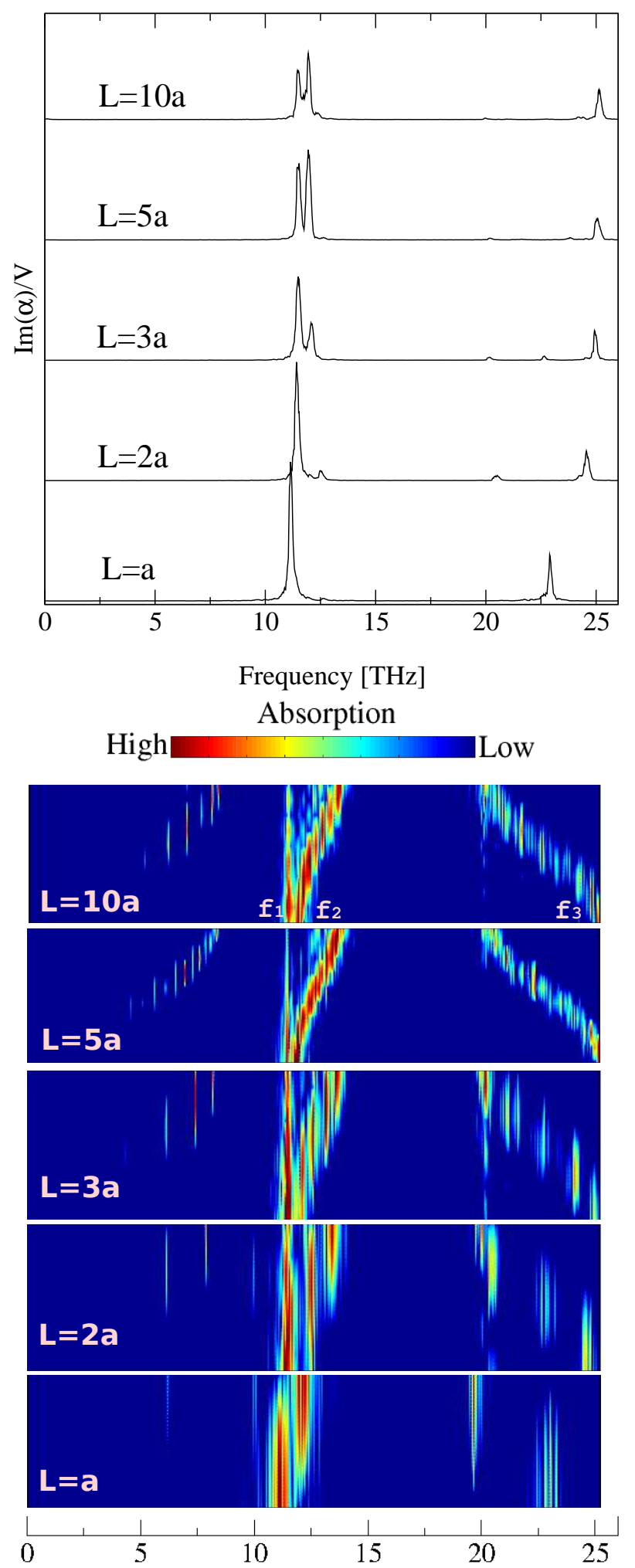

Frequency [THz]

FIG. 3. Size dependence of the imaginary part of the polarizability in $\mathrm{MgO}$ slabs ranging from $L=1 a$ to $L=10 a$. Top: Absorption of the slabs $(\mathbf{k}=0)$; Bottom: $\alpha^{\prime \prime}\left(\omega, \mathbf{k}_{\mathbf{z}}\right)$ in $\log$ scale

a Applied Physics Letter, 104(2014)011905. DOI: $10.1063 / 1.4860989$ 